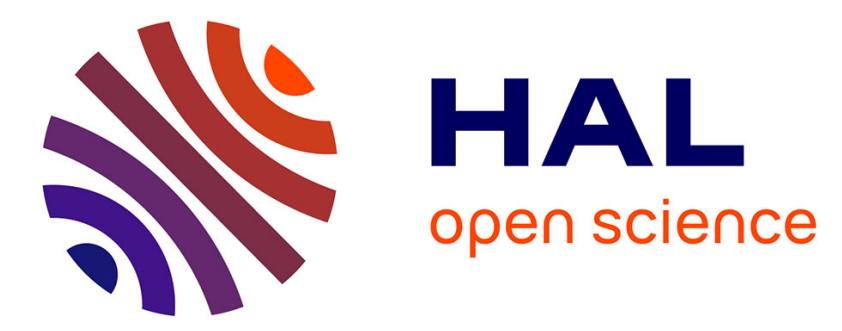

\title{
Charge Distribution and Contact Resistance Model for Coplanar Organic Field-Effect Transistors
}

\author{
Chang Hyun Kim, Yvan Bonnassieux, Gilles Horowitz
}

\section{To cite this version:}

Chang Hyun Kim, Yvan Bonnassieux, Gilles Horowitz. Charge Distribution and Contact Resistance Model for Coplanar Organic Field-Effect Transistors. IEEE Transactions on Electron Devices, 2013, 60 (1), pp.280. 10.1109/ted.2012.2226887 . hal-00770549

\section{HAL Id: hal-00770549}

https://hal-polytechnique.archives-ouvertes.fr/hal-00770549

Submitted on 7 Jan 2013

HAL is a multi-disciplinary open access archive for the deposit and dissemination of scientific research documents, whether they are published or not. The documents may come from teaching and research institutions in France or abroad, or from public or private research centers.
L'archive ouverte pluridisciplinaire $\mathbf{H A L}$, est destinée au dépôt et à la diffusion de documents scientifiques de niveau recherche, publiés ou non, émanant des établissements d'enseignement et de recherche français ou étrangers, des laboratoires publics ou privés. 


\title{
Charge Distribution and Contact Resistance Model for Coplanar Organic Field-Effect Transistors
}

\author{
Chang Hyun Kim, Student Member, IEEE, Yvan Bonnassieux, and Gilles Horowitz
}

\begin{abstract}
We propose a theoretical description of the charge distribution and the contact resistance in coplanar organic field-effect transistors (OFETs). Based on the concept that the current in organic semiconductors is only carried by injected carriers from the electrodes, an analytical formulation for the charge distribution inside the organic layer was derived. We found that the contact resistance in coplanar OFETs arises from a sharp low-carrier-density zone at the source/channel edge because the gate-induced channel carrier density is orders of magnitude higher than the source carrier density. This image is totally different from the contact resistance in staggered OFETs, in which the contact resistance mainly originates from the resistance through the semiconductor bulk. The contact resistance was calculated through charge-distribution functions and the model could explain the effect of the gate voltage and injection barrier on the contact resistance. Experimental data on pentacene OFETs were analyzed using the transmission-line method. We finally noticed that the gate-voltage dependent mobility is a critical factor for proper understanding of the contact resistance in real devices.
\end{abstract}

Index Terms

Charge distribution, contact resistance, coplanar OFETs, physical modeling.

\section{INTRODUCTION}

I $\mathrm{N}$ SPITE OF impressive advances in organic electronic devices, theoretical understanding of their operation is still incomplete. Organic field-effect transistors (OFETs), which are of great importance for low-cost flexible electronics, need more fundamental insights for further breakthroughs [1]. Among other interesting features of OFETs, contact resistance $\left(R_{c}\right)$ effect is decisive because there exists a substantial injection barrier in most realistic metal/organic junctions. In other words, even if one might choose a metal of which the Fermi level is very close to the transport orbital (HOMO or LUMO) of the organic material, process contamination and formation of interface trap states generally result in a Fermi level pinning so that the injection barrier cannot vanish below certain level [2], [3].

It is widely accepted that device geometry plays a crucial role for $R_{c}$ and comparative experiments on staggered and coplanar structures have been reported [4], [5]. Fig. 1 illustrates four common device geometries of OFETs. Typically, staggered OFETs showed smaller $R_{c}$ than coplanar OFETs. This tendency was tentatively explained by various concepts including metal penetration into organic films [6] and lateral morphological variation of polycrystalline film [5], [7]. In both geometries, $R_{c}$ seemed to be also dependent on the applied gate voltage $\left(V_{G}\right)$ with its magnitude decreasing with increasing $\left|V_{G}\right|$. Several models based on the current crowding mechanism have been invoked to explain the $V_{G}$ dependence of $R_{c}$ in staggered OFETs [8], [9], [10], [11]. However, no such consummate operation model exists for the origin and the $V_{G}$-dependence of $R_{c}$ in coplanar OFETs. The coplanar structure is more frequently adopted than the staggered one in fabrication. This is due to the fact that people prefer to avoid metal-deposition damage on a 'fragile' organic film in bottom-gate configuration. Therefore, a specific model for coplanar OFETs is highly desired. Concerning the geometrical effect on $R_{c}$, we recently observed that, apart from the above-mentioned process-related factors, there is a fundamental difference in the 'charge distribution' in staggered and coplanar organic transistors and this fact implies an underlying physical background of the dissimilar contact behaviors [12].

Here, we propose a physical device model for coplanar OFETs. Analytical equations for the two-dimensional charge distribution are developed by solving Poisson's and transport equations and by applying boundary conditions for a finitethickness semiconductor. The carrier-density in the transition zone at the source/channel interface is modeled to build up a formulation of $R_{c}$ as an integration of the local resistivity. Numerically calculated $R_{c}$ clearly shows the influence of $V_{G}$ and injection barrier height $\left(E_{b}\right)$ on the determination of $R_{c}$. Experimental data on coplanar OFETs are analyzed by testing coplanar pentacene transistors. By applying the transmission-line method (TLM) to the measurement results, it is revealed that the $V_{G}$-dependence of mobility is another pivotal factor that should be taken into account.

\section{EXPERIMENTAL AND MODELING METHODS}

Pentacene-based coplanar OFETs were fabricated with the structure equivalent to the the model transistor in Fig. 2. The bottom-gate electrode was prepared by a deposition of $\mathrm{Cr}$ on a glass substrate. A chemically and mechanically stable epoxybased photoresist SU-8 was used as a gate insulator. A diluted SU-8 solution (SU-8 2050 product of MicroChem) was

The authors are with the Laboratoire de Physique des Interfaces et des Couches Minces (CNRS UMR-7647), Ecole Polytechnique, 91128 Palaiseau, France. (e-mail: chang-hyun.kim@ polytechnique.edu; yvan.bonnassieux@ polytechnique.edu; gilles.horowitz@ polytechnique.edu) 

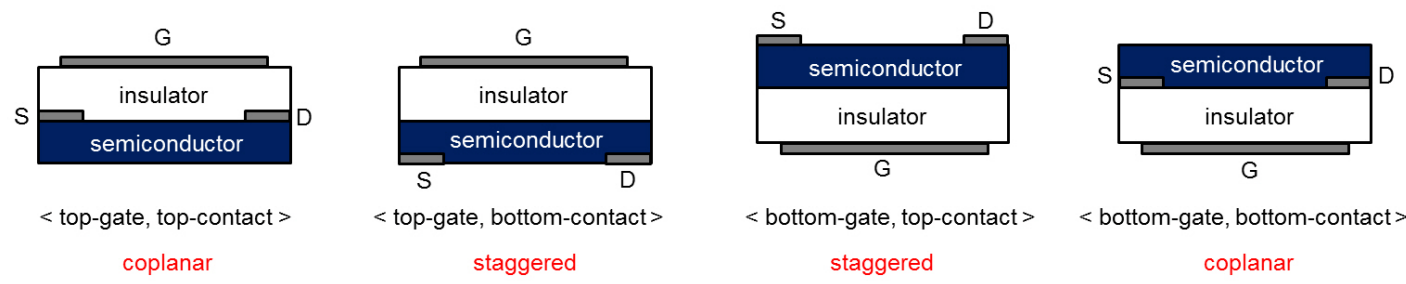

Fig. 1. Four OFET structures characterized by the relative positions of the gate $(\mathrm{G})$ and the source/drain (S/D) electrodes. By definition, the source/drain electrodes in coplanar OFETs are on the same plane with the conducting channel. In the staggered structure, on the contrary, the semiconducting layer separates the source/drain electrodes from the channel. The model presented in this study is applicable to the two coplanar structures.

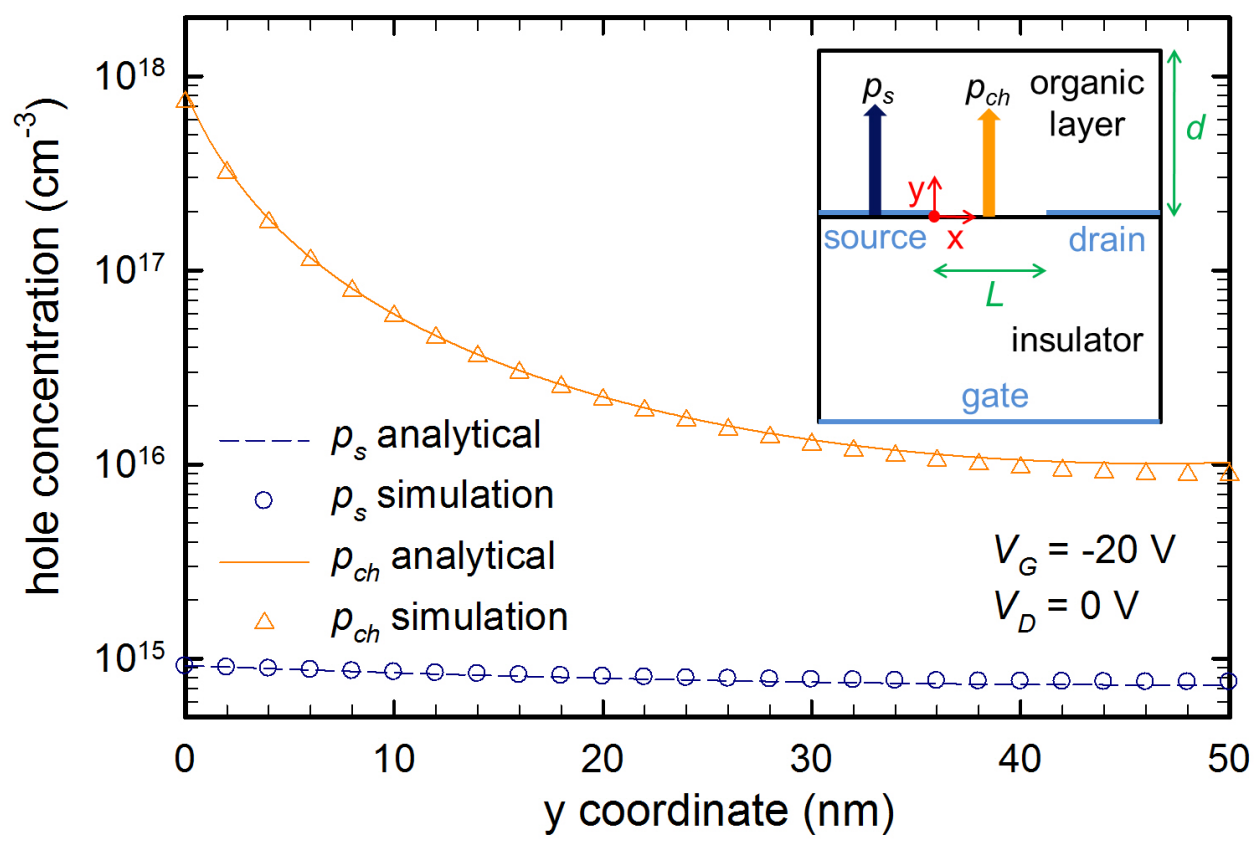

Fig. 2. Charge distribution inside the organic semiconductor of a coplanar-type OFET. The analytical curves are obtained by (15), (17), (23), and (27). Note that the device simulator brings very close results.

spin-coated on the substrate and UV-exposed to activate the cross-linking reaction. The processed film was hard-baked at 200 ${ }^{\circ} \mathrm{C}$ for 2 minutes. This method gave a $950 \mathrm{~nm}$-thick polymeric film with the gate capacitance of $2.3 \mathrm{nF} / \mathrm{cm}^{2}$. The source/drain electrodes were then formed by depositing $\mathrm{Au}$ followed by a photolithographical patterning step. Pentacene (99.9+\% purity, used as-recieved from Sigma-Aldrich) was finally vacuum-evaporated to form a hole-trasporting molecular film. During the thermal evaporation of pentacene, the substrate was heated at $50{ }^{\circ} \mathrm{C}$ and the deposition rate was kept at $0.1 \mathrm{~nm} / \mathrm{s}$ with the final thickness of $50 \mathrm{~nm}$. We have recently estimated the quasistatic dielectric constant of pentacene in [13] and the average value of 3.6 will be used in this study.

Current-voltage $(I-V)$ characteristics were measured using a semiconductor characterization system (Keithley 4200) in the dark under ambient atmosphere. Tapping-mode atomic force microscopy (AFM) images of the pentacene film were taken using Veeco Dimension 5000 AFM system. Model calculations were performed with MATHCAD platform (Parametric Technology Corporation). To support the model, simulation results were taken through two-dimensional physically-based ATLAS simulator (SILVACO). This finite-element computer simulator numerically solves a set of coupled Poisson's, continuity, and drift-diffusion equations within a user-defined two-dimensional mesh.

\section{CHARGE-CARRIER DISTRIBUTION IN AN ORGANIC SEMICONDUCTOR}

The aim of this section is to find how the injected holes from the source $\left(p_{s}\right)$ and the gate-induced holes at the channel $\left(p_{c h}\right)$ are distributed along the thickness of the organic layer ( $y$-axis) in the model transistor depicted in Fig. 2. Throughout this study, a two-dimensional model will be developed based on the following hypotheses: 1) All charge carriers are "injected" into the organic semiconductor; 2) hole-only conduction is considered; 3) the gradual channel approximation holds. We recently confirmed that unintentionally doped organic semiconductors are fully depleted so that the above assumption 1) can be made [13]. By this simplification, an organic semiconductor is rather an insulator that can only 'transport' the carriers given by external circumstances (injection or illumination) and we can revisit the classical theory on the metal/insulator junction that 
has been intensively dealt with in the early days of solid-state electronics through the 1940s and 1950s. We will first briefly recapitulate traditional models in Section III-A and propose a new approach in Section III-B.

\section{A. Revisiting classical models}

Imagine a one-dimensional insulator (or undoped semiconductor) in contact with a charge reservoir (normally a metal electrode) at $y=0$ that extends toward the positive $y$-direction up to $y=d$ ( $d$ is the thickness of the semiconductor). The electrostatic distribution of injected carriers $p(y)$ can be estimated by simultaneously analyzing Poisson's equation (1) and the transport (drift-diffusion) equation (2)

$$
\begin{gathered}
\frac{\mathrm{d} F}{\mathrm{~d} y}=\frac{q p(y)}{\epsilon_{s}}, \\
J_{p}=q p \mu F-q D \frac{\mathrm{d} p}{\mathrm{~d} y},
\end{gathered}
$$

where $F(y)$ is the electric field, $q$ the elementary charge, $p(y)$ the hole concentration, $\epsilon_{s}$ the permittivity of the semiconductor, $J_{p}$ the net hole current density, $\mu$ the hole mobility, and $D$ the hole diffusion coefficient. These two equations are then merged by substituting $p$ in (2) using (1) and by taking Einstein relation $D / \mu=k T / q$, where $k$ is the Boltzmann constant and $T$ the absolute temperature. This gives

$$
J_{p}=\epsilon_{s} \mu\left(F \frac{\mathrm{d} F}{\mathrm{~d} y}-\frac{k T}{q} \frac{\mathrm{d}^{2} F}{\mathrm{~d} y^{2}}\right),
$$

which is the fundamental equation of the given physical system to be solved. At thermal equilibrium, the net current is zero (the drift current and the diffusion current compensate each other) so that

$$
F \frac{\mathrm{d} F}{\mathrm{~d} y}-\frac{k T}{q} \frac{\mathrm{d}^{2} F}{\mathrm{~d} y^{2}}=0
$$

This can be integrated once to

$$
\left(\frac{q}{2 k T}\right)^{2} F^{2}-\frac{q}{2 k T} \frac{\mathrm{d} F}{\mathrm{~d} y}=-g^{2}
$$

where $g$ is an integration constant.

Mott and Gurney first derived the solution of (5) for a semi-infinite semiconductor $(d \rightarrow \infty)$ [14]. In this case, both $F(y)$ and $\mathrm{d} F / \mathrm{d} y$ go to zero when $y \rightarrow \infty$, so that $g=0$ and (5) reduces to

$$
F^{2}-\frac{2 k T}{q} \frac{\mathrm{d} F}{\mathrm{~d} y}=0
$$

The solution for the electric field is reached by separating variables,

$$
F(y)=\left(-\frac{2 k T}{q y_{0}}\right)\left(\frac{1}{1+y / y_{0}}\right)=\frac{F_{0}}{1+y / y_{0}},
$$

where $y_{0}$ is another integration constant. The hole distribution $p(y)$ is given by the first derivative of $F(y)$;

$$
p(y)=\left(\frac{2 \epsilon_{s} k T}{q^{2} y_{0}^{2}}\right)\left(\frac{1}{1+y / y_{0}}\right)^{2}=\frac{p_{0}}{\left(1+y / y_{0}\right)^{2}} .
$$

$F_{0}$ and $p_{0}$ are respectively the electric field and the hole concentration at the junction $(y=0)$. Equations (7) and (8) are intended to make more visible the physical meaning of $y_{0}$, which can be viewed as a characteristic length over which the boundary value $\left(F_{0}\right.$ or $\left.p_{0}\right)$ is distributed from the junction interface. The value of $y_{0}$ can be determined from the boundary value of either $F_{0}$ or $p_{0}$. For the following discussion, a relationship between $y_{0}$ and $p_{0}$ is particularly useful. From (8), we have

$$
y_{0}=\sqrt{\frac{2 \epsilon_{s} k T}{q^{2} p_{0}}},
$$

meaning that the higher the initial carrier density $p_{0}$ is, the smaller $y_{0}$ becomes. If $y_{0}$ is small, the carriers are densely concentrated at the junction and do not spread far away from the injecting surface. Even though the Mott-Gurney model provides meaningful insight into the charge distribution, its usage should be limited to very thick organic crystals and this model cannot be safely applied to thin organic films.

Skinner stepped forward and developed more general solutions to (5) [15]. It means that one can challenge the 'finite' junction without forcing $g$ to zero. The author actually separated the cases by the sign of the integration constant and obtained separate sets of solutions depending on this sign. An essential boundary condition for the finite semiconductor is $F(d)=0$ because there cannot be any current flowing into or out of the semiconductor at the surface $y=d$. The injected holes (positive charges) make the only contribution to the space charge in Poisson's equation. Consequently, the sign of $\mathrm{d} F / \mathrm{d} y$ is always 
positive through the whole semiconductor thickness. In other words, $F(0)$ is negative and $F(y)$ approaches zero from $y=0$ to $y=d$. At $y=d, F$ becomes zero by the boundary condition and $\mathrm{d} F / \mathrm{d} y$ remains positive. Therefore, the right-hand term in (5) is negative and the corresponding solution is the trigonometric function in [15], which can be written in the form;

$$
\begin{gathered}
F(y)=-\frac{q p_{0} g y_{0}^{2}}{\epsilon_{s}} \cot \left(g y+\arcsin g y_{0}\right), \\
p(y)=\frac{p_{0} g^{2} y_{0}^{2}}{\sin ^{2}\left(g y+\arcsin g y_{0}\right)} .
\end{gathered}
$$

Here, $p_{0}$ always represents the hole concentration at $y=0$ and (9) remains valid. In order to estimate the constant $g$, we introduce the boundary condition $F(d)=0$. From (10), this gives

$$
\cot \left(g d+\arcsin g y_{0}\right)=0
$$

which can be further simplified by using trigonometric identities, resulting in

$$
g y_{0}=\cos g d
$$

By putting (13) into (11), we can write

$$
p(y)=\frac{p_{0} \cos ^{2} g d}{\cos ^{2} g(d-y)},
$$

where $0 \leq y \leq d$.

A limitation of Skinner's approach looks apparent at this point. Although this model gives exact solutions for thin-film cases, (13) does not lead to an analytical expression for $g$ and, consequently, the final solution requires numerical computation.

\section{B. Approximate solutions}

Now let us return to the two-dimensional semiconductor and discuss how the initial carrier densities at the insulator/semiconductor interface $(y=0)$ are determined in the coplanar OFET architecture (see the inset in Fig. 2). The carrier density at a metal/semiconductor interface is dictated by Boltzmann's statistics [16] so that $p_{s 0}$ (the source carrier density at $y=0$ ) is strongly injection-limited following

$$
p_{s 0}=N_{v} \exp \left(-\frac{E_{b}}{k T}\right),
$$

where $N_{v}$ is the effective density of states at the HOMO edge. The hole barrier height $E_{b}$ corresponds the energy between the electrode Fermi level and the semiconductor HOMO level. The channel carriers, on the other hand, are induced by the gate capacitance and can be estimated by

$$
Q_{c h}=C_{i}\left|V_{G}-V_{T}\right|=q \int_{0}^{d} p_{c h}(y) \mathrm{d} y,
$$

where $Q_{c h}$ is the total channel charge per unit area, $C_{i}$ the insulator capacitance per unit area, and $V_{T}$ the threshold voltage. The functional form of $p_{c h}(y)$ will be derived below but it is helpful to know the boundary value already here. The channel hole concentration at $y=0$ is approximate to

$$
p_{c h 0} \approx \frac{Q_{c h}^{2}}{2 \epsilon_{s} k T}=\frac{C_{i}^{2}\left|V_{G}-V_{T}\right|^{2}}{2 \epsilon_{s} k T} .
$$

The characteristic distribution lengths for the source and the channel charges are then defined by (9), thus $y_{s 0}=\sqrt{2 \epsilon_{s} k T / q^{2} p_{s 0}}$ and $y_{c h 0}=\sqrt{2 \epsilon_{s} k T / q^{2} p_{c h 0}}$ respectively.

We can infer from (15) and (17) that $E_{b}$ and $V_{G}$ are the principal parameters for the source and channel distribution of charges. The results presented in Fig. 3 support this statement and give another important implication for the modeling. Fig. 3 shows the results calculated by (15) and (17) with $N_{v}=10^{20} \mathrm{~cm}^{-3}, T=300 \mathrm{~K}, \epsilon_{s}=3.6 \times \epsilon_{0}, C_{i}=2.3 \mathrm{nF} / \mathrm{cm}^{2}$, and $V_{T}=0 \mathrm{~V}\left(\epsilon_{0}\right.$ is the permittivity of vacuum). Typical organic materials exhibit a transport band width of around 500 meV, which is much larger than the thermal energy $k T$. In this case, $N_{v}$ should be lower than the total molecular denisity and $10^{20} \mathrm{~cm}^{-3}$ is a realistic value for $N_{v}$. At variance with the charge distribution in staggered OFETs in which the gate-induced charges are evenly distributed over the whole semiconductor/insulator interface [12], here $p_{s 0}$ is much lower than $p_{c h 0}$ due to the high injection barrier. Another key feature of Fig. 3 is that $y_{s 0}$ normally exceeds the thickness of an organic thin-film, whereas $y_{c h 0}$ is far smaller than the film thickness. This finding enables an independent modeling of $p_{s}(y)$ and $p_{c h}(y)$ by means of an approximation method.

The main idea is to develop an analytical form of (14) by approximating the cosine function in (13). A linear (or a first-order) approximation of any given function $f(z)$ is defined at the vicinity of $z=a$ by

$$
f(z) \approx f(a)+f^{\prime}(a)(z-a) .
$$



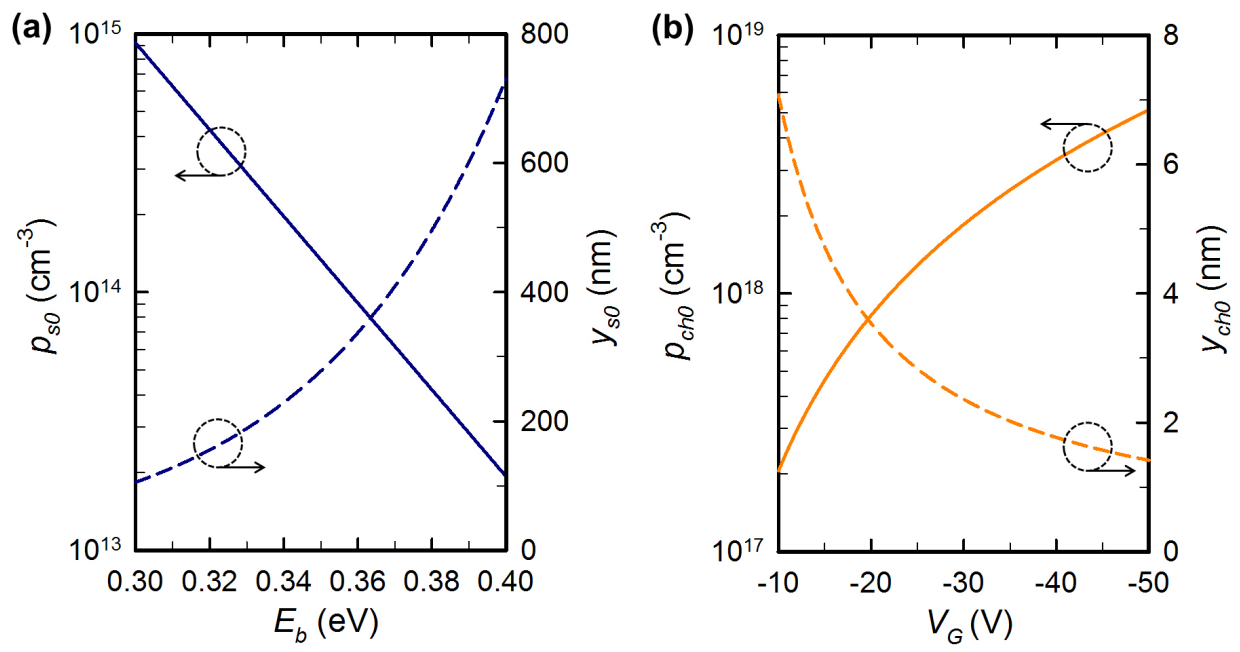

Fig. 3. (a) Source distribution factors $\left(p_{s 0}\right.$ and $\left.y_{s 0}\right)$ as a function of $E_{b}$ and (b) channel distribution factors $\left(p_{c h 0}\right.$ and $\left.y_{c h 0}\right)$ as a function of $V_{G}$. The solid lines indicate the initial hole concentrations and the dashed lines correspond to the distribution lengths. Note how different the order of the vertical axis is in plot (a) and (b).

If $f(z)=\cos z$, we get

$$
\cos z \approx \cos a+(-\sin a)(z-a) .
$$

First, for the source carriers $p_{s}(y)$, we rewrite (13) as

$$
g_{s} y_{s 0}=\cos g_{s} d
$$

where the subscript ' $s$ ' refers to the 'source'. Because $y_{s 0} \gg d$, we can approximate $\cos g_{s} d$ where $g_{s} d$ is close to zero. Under this condition, we can say

$$
g_{s} y_{s 0}=\cos g_{s} d \approx 1
$$

and therefore

$$
g_{s} \approx \frac{1}{y_{s 0}}
$$

Then if we use (22) to replace $g$, (14) finally changes into

$$
p_{s}(y)=p_{s 0} \frac{\cos ^{2}\left(\frac{d}{y_{s 0}}\right)}{\cos ^{2}\left(\frac{d-y}{y_{s 0}}\right)} .
$$

Next, another approximation is needed for the channel carriers $p_{c h}(y)$. Starting again from (13),

$$
g_{c h} y_{c h 0}=\cos g_{c h} d,
$$

where the subsrcipt ' $c h$ ' refers to the 'channel'. This time, $y_{c h 0} \ll d$ so that $\cos g_{c h} d$ should be developed near $g_{c h} d=\pi / 2$. Referring to (19), we get

$$
g_{c h} y_{c h 0}=\cos g_{c h} d \approx \frac{\pi}{2}-g_{c h} d
$$

and

$$
g_{c h} \approx \frac{\pi}{2\left(d+y_{\operatorname{ch} 0}\right)} .
$$

The channel carrier distribution function $p_{c h}(y)$ is now given from (14) and (26), after some manipulation steps, by

$$
p_{c h}(y)=p_{\operatorname{ch} 0} \frac{\sin ^{2}\left(\frac{\pi}{2} \frac{y_{c h 0}}{d}\right)}{\sin ^{2}\left(\frac{\pi}{2} \frac{y_{c h 0}+y}{d}\right)} .
$$

Now one can reexamine the integration in (16) and see that (17) is correct under the condition that $y_{\text {ch } 0} \ll d$.

It is worth emphasizing here that our approximate model [(23) and (27) with (15) and (17)] provides analytical expressions that explicitly contain the thickness parameter $d$. It means that this strategical development overcomes the limitation of the two classical models summarized in Section III-A and assures its general applicability to the thin film-based OFETs. Fig. 2 shows the reliability of the approximate solutions. The parameters used here in both the analysis and the simulation are those listed above for Fig. 3 with $d=50 \mathrm{~nm}, E_{b}=0.3 \mathrm{eV}$, and $V_{G}=-20 \mathrm{~V}$. It is shown that the analytical model predicts the numerical simulation results with satisfying precision. 


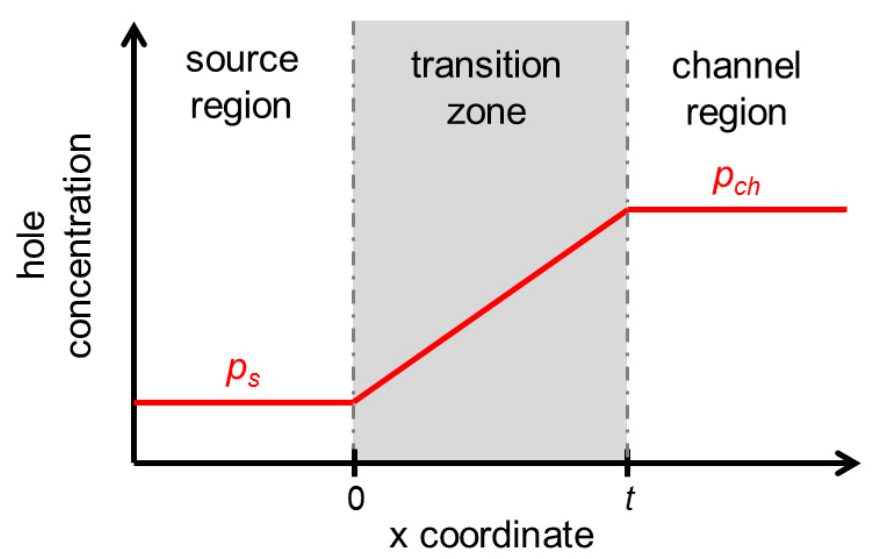

Fig. 4. A conceptual representation for the hole concentration near the transition zone that is located from $x=0$ to $x=t$. As explained in Section IV-A, the $x$-directional change is modeled by an exponential function so the vertical axis in the figure is in logarithmic scale.

\section{Charge-BASED CONTACT RESISTANCE MODEL}

Now that we have a two-dimensional picture of the charge distribution inside the organic film in coplanar OFETs, we develop a charge-based contact resistance model in this section. Section IV-A will deal with a mathematical formulation of $R_{c}$ in the transition zone and the effect of the critical parameters will be discussed in Section IV-B

\section{A. Resistance of the transition zone}

We realize that there is an abrupt transition of the hole concentration at $x=0$ due to the huge difference between $p_{s}(y)$ and $p_{c h}(y)$. It is this carrier-density transition zone that accounts for the origin of $R_{c}$ because the hole concentration in this zone is much lower than that in the conducting channel, due to the effect of $p_{s}$ penetrating into the channel region. Now let us contemplate what happens at this source/channel interface where two independent distribution functions overlap (Fig. 4). There exist concentration tails along the $x$-direction characterized by the Debye length of the channel carriers $\left(x_{c h}\right)$ and that of the source carriers $\left(x_{s}\right)$. They are defined by $x_{c h}=\sqrt{\epsilon_{s} k T / q^{2} p_{c h}}$ and $x_{s}=\sqrt{\epsilon_{s} k T / q^{2} p_{s}}[16]$. Because $p_{c h} \gg p_{s}, x_{c h} \ll x_{s}$ and we can neglect the contribution of $x_{c h}$ so that the the transition from $p_{s}$ to $p_{c h}$ can be simplified to a single exponential function

$$
p(x)=p_{s} \exp \left(\frac{x}{x_{s}}\right)
$$

Then the thickness $t$ of the transition zone in Fig. 4 is estimated by

$$
\begin{gathered}
p(t)=p_{s} \exp \left(\frac{t}{x_{s}}\right)=p_{c h}, \\
t=x_{s} \ln \left(\frac{p_{c h}}{p_{s}}\right) .
\end{gathered}
$$

Here, it is worth mentioning that $t$ also varies along the semiconductor thickness as $p_{c h}$ and $p_{s}$ change together along the $y$-direction. Taking the parameters used in Section III-B, the calculated $t$ is $0.51 \mu \mathrm{m}$ at $y=0$. It means that only a narrow region of the entire channel near the source electrode is affected by low $p_{s}$.

The contact resistance $R_{c}$ is calculated from an estimation of the average hole concentration in the transition zone (or $R_{c}$ zone), done by integrating the local conductivity over the entire thickness. Keeping this approach in mind, the mean hole concentration $p_{m}$ throughout the $R_{c}$ zone can be defined by

$$
p_{m}=\frac{1}{t} \int_{0}^{t} p(x) \mathrm{d} x=\frac{p_{s}}{t} \int_{0}^{t} \exp \left(\frac{x}{x_{s}}\right) \mathrm{d} x,
$$

where (28) is used. By inserting (29) and (30) and using $p_{c h} \gg p_{s}$, we get

$$
p_{m}=\frac{p_{c h}-p_{s}}{\ln \left(p_{c h} / p_{s}\right)} \approx \frac{p_{c h}}{\ln \left(p_{c h} / p_{s}\right)},
$$

which shows that the balance between $p_{s}$ and $p_{c h}$ determines the average concentration and $p_{m}$ is also a function of $y$. Now we can calculate the elemental conductance $\mathrm{d} G_{c}$ of the volume element delimited by $W$ (channel width), $t(y)$, and $\mathrm{d} y$

$$
\mathrm{d} G_{c}=q \mu W \frac{p_{m}(y)}{t(y)} \mathrm{d} y
$$




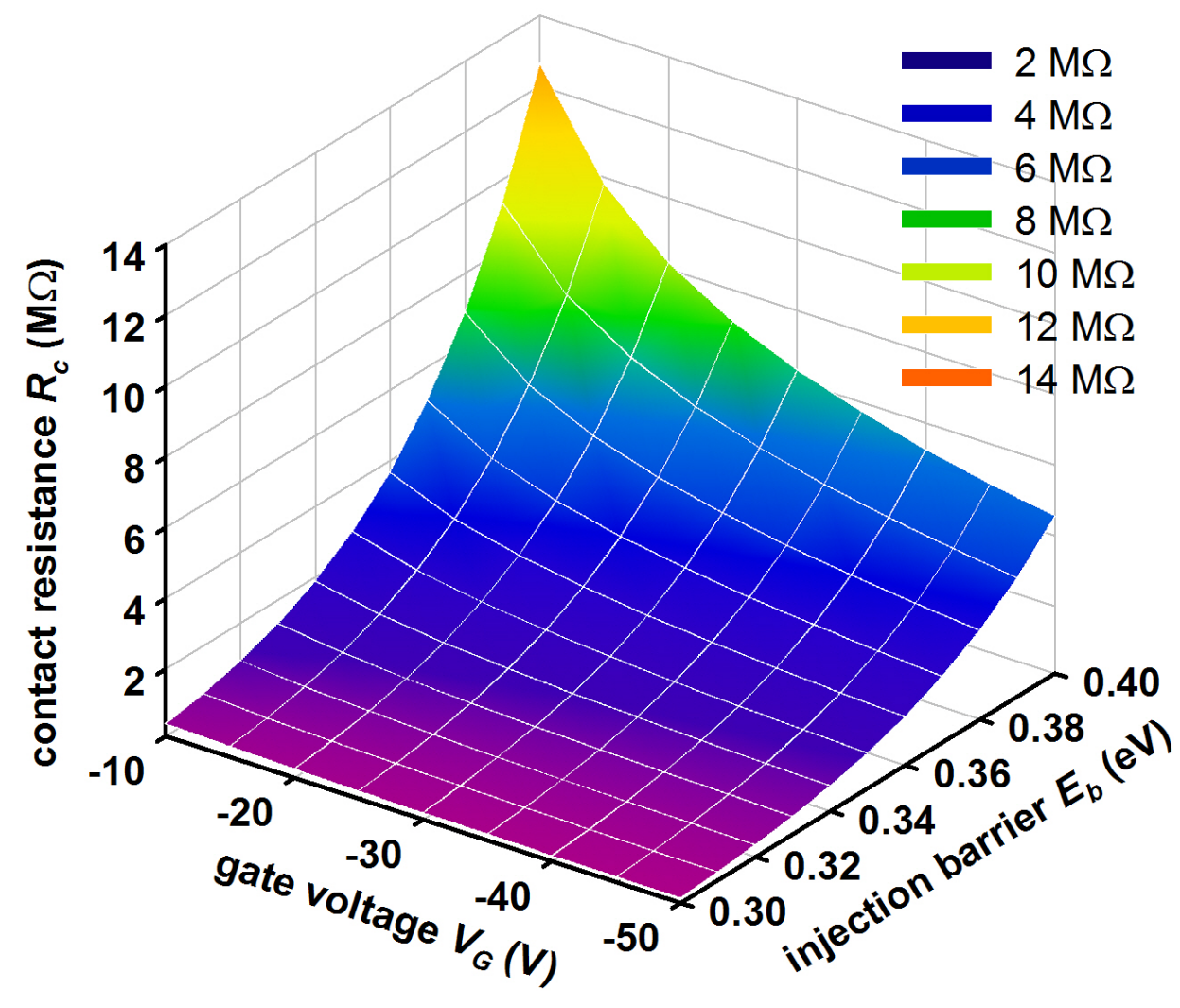

Fig. 5. $R_{c}$ determined by (34) with a variation of $V_{G}$ and $E_{b}$. The fixed parameters are as follows; $N_{v}=10^{20} \mathrm{~cm}^{-3}, T=300 \mathrm{~K}, \epsilon_{s}=3.6 \times \epsilon_{0}$, $C_{i}=2.3 \mathrm{nF} / \mathrm{cm}^{2}, V_{T}=0 \mathrm{~V}, d=50 \mathrm{~nm}, W=500 \mu \mathrm{m}$, and $\mu=0.2 \mathrm{~cm}^{2} / \mathrm{V} \cdot \mathrm{s}$.

where the hole mobility $\mu$ is assumed to be constant at this moment. $R_{c}$ is finally obtained, by replacing $t(y)$ and $p_{m}(y)$ using (30) and (32),

$$
R_{c}=\frac{1}{G_{c}}=\frac{\sqrt{\epsilon_{s} k T}}{q^{2} \mu W}\left[\int_{0}^{d} \frac{p_{c h}(y) \sqrt{p_{s}(y)}}{\left[\ln \left(p_{c h}(y) / p_{s}(y)\right)\right]^{2}} \mathrm{~d} y\right]^{-1} .
$$

Note that, from (34), it is possible to predict the exact value of $R_{c}$ when the distribution functions for $p_{s}(y)$ and $p_{c h}(y)$ are known.

\section{B. Effect of the parameters}

Numerically calculated $R_{c}$ values are plotted in Fig. 5 with varying $V_{G}$ and $E_{b}$. The charge-distribution functions $\left[p_{s}(y)\right.$ and $p_{c h}(y)$ ] developed in Section III-B were inserted to (34). All fixed parameters are summarized in the caption to Fig. 5. We can state, from this result, that the theoretical charge-based model well predicts the decrease of $R_{c}$ with increasing $\left|V_{G}\right|$, which is often experimentally observed, though not sufficiently understood in the case of coplanar OFETs [17], [18], [19], [20]. Furthermore, it can be inferred that the degree of dependency between $V_{G}$ and $R_{c}$ is accentuated with higher injection barriers. Finally, it should be noted that the hole mobility $\mu$ is considered constant here, which restricts the analysis to defect-free, highly pure crystalline semiconductors [21]. When substantial trap states exist, $\mu$ should be regarded as the 'effective' mobility determined by the ratio of free to total carrier density. This results in the dependence of the measured mobility on the gate voltage as will be discussed in the next section.

\section{APPLiCATION TO EXPERIMENTAL RESUlTS}

This section is dedicated to the analysis of experimental results on fabricated OFETs with the $R_{c}$ model. In Section V-A, the basic electrical properties of pentacene-based OFETs will be presented. Then, in Section V-B, we will concisely summarize how the transmission-line method works for experimentally extracting $R_{c}$. Finally, the $V_{G}$-dependent mobility and its influence on $R_{c}$ will be discussed in Section V-C. 

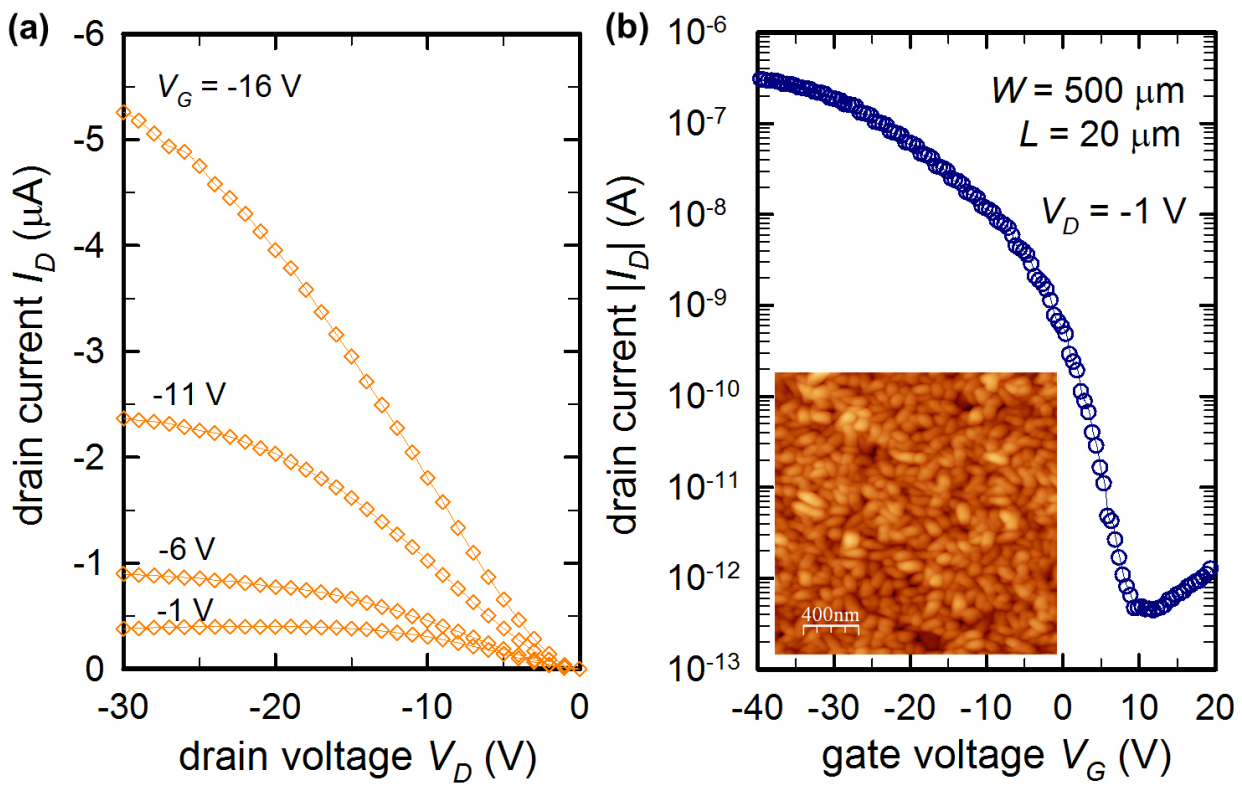

Fig. 6. Measured electrical performance of a pentacene transistor fabricated with the bottom-gate, bottom-contact geometry. (a) Output characteristics and (b) transfer characteristics. The inset of (b) is an AFM image taken on the $50 \mathrm{~nm}$-thick pentacene film in the channel region (scan size: $2 \times 2 \mu \mathrm{m}^{2}$ ).

\section{A. Output and transfer curves}

The electrical performance of a representative transistor is shown in Fig. 6. The channel width $W$ and length $L$ of this transistor are $500 \mu \mathrm{m}$ and $20 \mu \mathrm{m}$ respectively. The slight upward bending of the output curves [Fig. 6(a)] at low drain voltage $\left(V_{D}\right)$ is direct evidence for a non-linear parasitic contact effect [18]. Hence, it can be predicted that there is non-negligible contribution of $R_{c}$ to the current in the fabricated device. A linear-regime transfer curve is shown in Fig. 6(b). One can see that the off-state current is extremely low and close to the detection limit of the measurement system (10 ${ }^{-12}$ A), assuring an excellent gate-insulation by the SU-8 film. The AFM image in Fig. 6(b) shows relatively small domain size of the order of $100 \mathrm{~nm}$. These small and uniform grains could be attributed to the three-dimensional growth of pentacene which contrasts with the two-dimensional layer-by-layer growth that results in large, dendritic grains [22].

\section{B. Transmission-line method}

The transmission-line method (TLM) is a widely-used technique for the extraction of $R_{c}$ from $I$ - $V$ data [23], [24], [25]. It is assumed that the source-to-drain current path is equivalent to a series combination of contact resistance $R_{c}$ and channel resistance $R_{c h}$ (inset in Fig. 7). The TLM is also strongly based on the assumption that $R_{c}$ is not a function of $L$ but $R_{c h}$ is proportional to $L$ [26]. At low $V_{D}$ (linear regime), the relationship between $I_{D}$ and $V_{D}$ of this circuit can be expressed as

$$
I_{D}=\frac{V_{D}}{R_{o n}}=\frac{V_{D}}{R_{c}+R_{c h}}=\frac{V_{D}}{R_{c}+\left[\frac{W}{L} \mu C_{i}\left|V_{G}-V_{T}\right|\right]^{-1}},
$$

where the linear-regime channel conductance is used to estimate $R_{c h} . R_{o n}$ is the on-state resistance that can be directly obtained from measured $V_{D}$ and $I_{D}$ by

$$
R_{o n}=\frac{V_{D}}{I_{D}}=R_{c}+\frac{L}{W \mu C_{i}\left|V_{G}-V_{T}\right|} .
$$

Width-normalized resistance is more practical for the purpose of comparing device sets with different $W$ so it is useful to multiply (36) by $W$ and get

$$
R_{o n} \times W=R_{c} \times W+\frac{L}{\mu C_{i}\left|V_{G}-V_{T}\right|} .
$$

To apply the TLM, we first draw $R_{o n} W$ versus $L$ and extrapolate the linear regression line to zero $L$ to read the $R_{c} W$ value. Fig. 7 shows the experimental data on pentacene OFETs. The deviation of the data points from the regression lines is minimal and it makes sure that the fabricated OFETs well satisfy the basic assumptions of the TLM. It is clear that $R_{c}$ decreases with increasing $\left|V_{G}-V_{T}\right|$ as the intercept to the vertical axis moves downward. $V_{T}$ for those three transistors were evaluated from the saturation current and no significant variation was observed so that we can use an average $V_{T}$ of $-4 \mathrm{~V}$. Although not often realized, it is important to note here that the TLM can also be used to estimate $\mu$ as a function of $\left|V_{G}-V_{T}\right|$ because the 'slope' of the regression line usually varies with $V_{G}$ (see Fig. 7) and this slope contains the parameter $\mu$ by (37). 


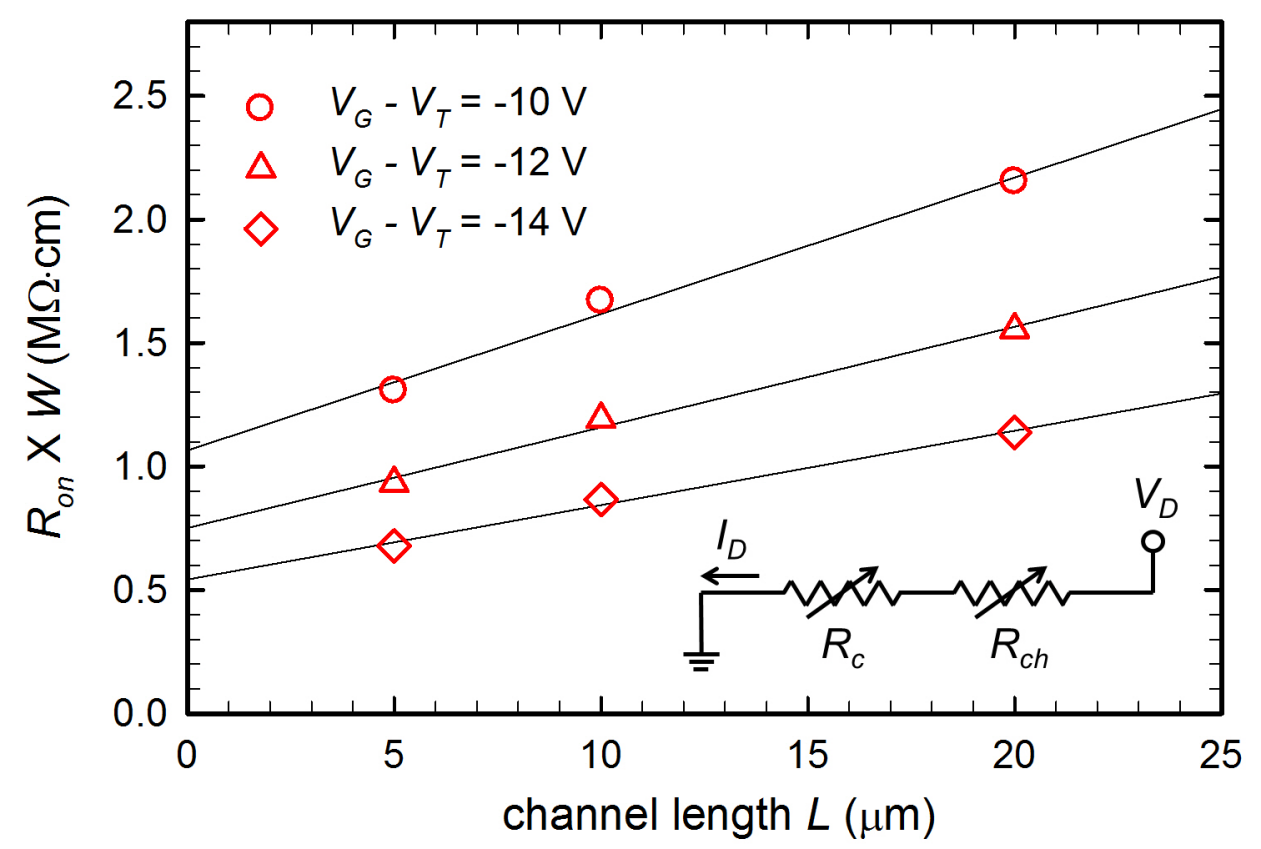

Fig. 7. $R_{\text {on }} W$ versus $L$ plot for the transmission-line method (TLM). The inset is the equivalent circuit of the on-state conduction path that consists of a series connection of the contact and channel resistances. Note that $R_{c}$ and $R_{c h}$ are drawn as variable resistors because both of them are supposed to be $V_{G}$-controlled.

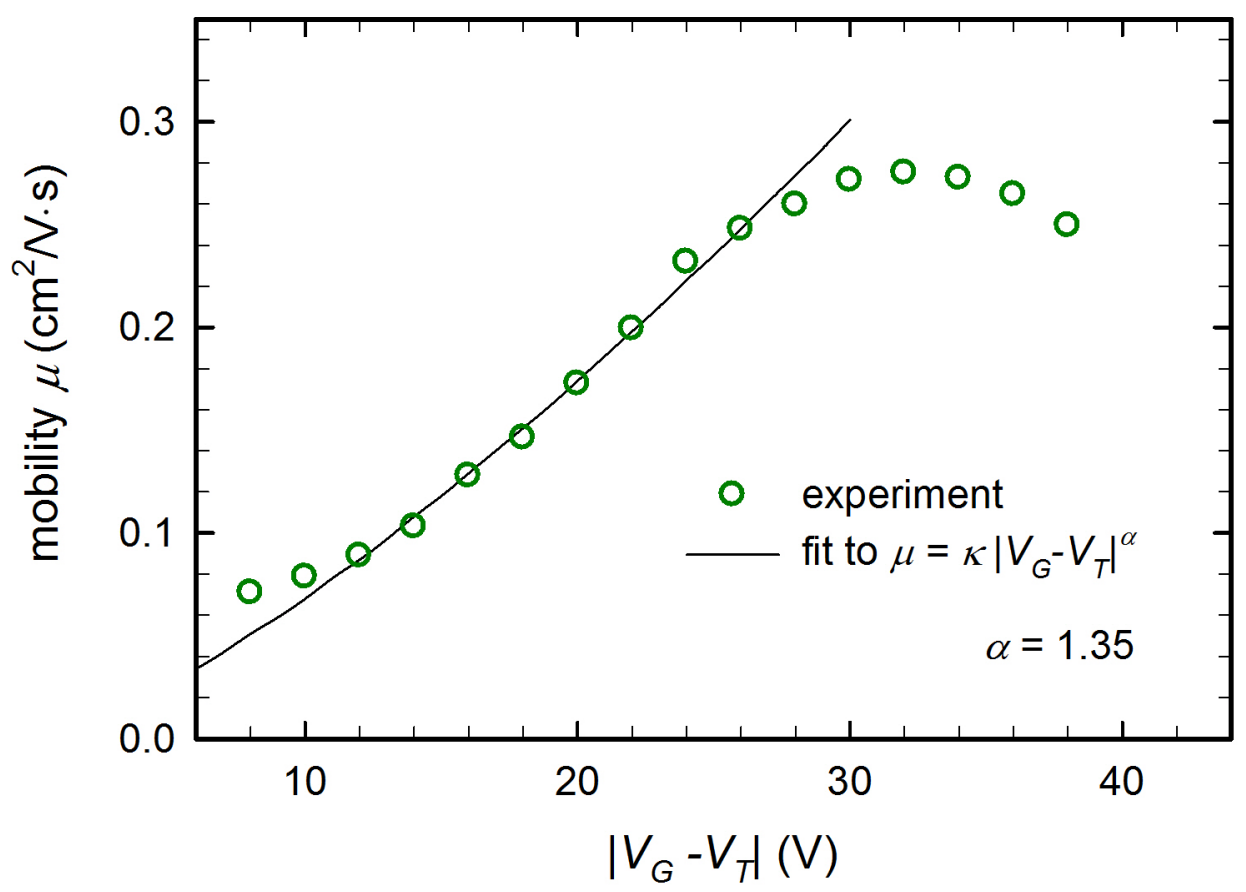

Fig. 8. Hole mobility as a function of $\left|V_{G}-V_{T}\right|$ extracted by the TLM. The solid line is a fit to the trap-filling model at low $\left|V_{G}\right|$. The decrease in the mobility at higher $\left|V_{G}\right|$ can be attributed to the field-induced mobility degradation.

\section{Effect of the gate-voltage dependent mobility}

The extracted hole mobility $\mu$ as a function of $\left|V_{G}-V_{T}\right|$ is shown in Fig. 8, which indicates that the variation of $\mu$ is significant and does not follow a simple monotonous behavior. We infer that the two distinct regimes are dominated by two different mechanisms. First, the rise in $\mu$ at low $\left|V_{G}\right|$ can be adequately described by the gradual filling of trap states as the Fermi level at the semiconductor/insulator interface approaches the transport orbital (HOMO in the case of p-type materials). This is reasonable because a polycrystalline pentacene film contains a large number of trapping sites, most of which located at the grain boundaries [27], [28]. We refer to the model of Horowitz et al. [29], [26] and fit the mobility in this regime 


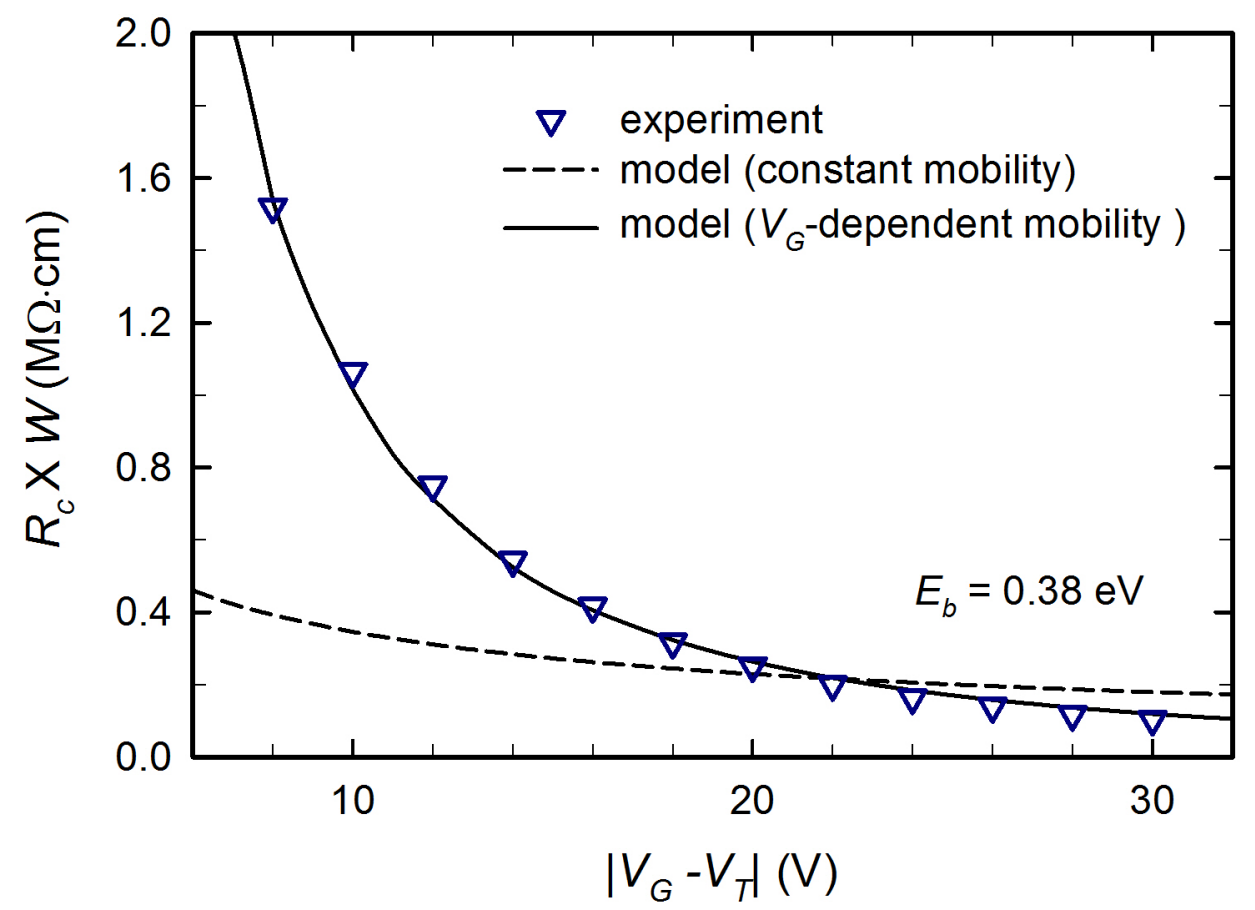

Fig. 9. TLM-extracted $R_{c} W$ as a function of $\left|V_{G}-V_{T}\right|$ in comparison to the charge-based model developed in Section IV. Note that, if a constant mobility is assumed (here $0.2 \mathrm{~cm}^{2} / \mathrm{V} \cdot \mathrm{s}$ ), the $V_{G}$-dependence of $R_{c}$ is underestimated. When we take the $V_{G}$-dependent mobility into account, the experimental results could be well fitted by the model. This method also enables an estimation of the injection barrier $E_{b}$.

$\left(\left|V_{G}-V_{T}\right|<30 \mathrm{~V}\right)$ to the equation

$$
\mu=\kappa\left|V_{G}-V_{T}\right|^{\alpha} .
$$

This model is based on the MTR (multiple trapping and release) process with an exponential density of states (DOS) near the band edge. The parameters $\kappa$ and $\alpha$ are then related to the trap DOS. The exponent $\alpha$ is directly linked to the trap characteristic temperature $T_{c}$ by $\alpha+1=\left(T_{c} / T\right)$. The extracted $\alpha$ is $1.35 \mathrm{in} \mathrm{Fig.} 8$ and the corresponding $T_{c}$ is $705 \mathrm{~K}$. If we convert this value into energy scale, the trap distribution width $E_{t}=k T_{c}$ is $61 \mathrm{meV}$, which is in good agreement with a recent comprehensive review on the trap DOS in pentacene field-effect transistors [30]. Next, the slight decrease in $\mu$ at higher $\left|V_{G}-V_{T}\right|$ can be interpreted by the field-induced mobility degradation [22]. It is likely that the mobility near the insulator surface is lower than that at the bulk region due to various surface scattering agents. When $\left|V_{G}\right|$ becomes high enough, field-induced holes are more concentrated at this low-mobility near-insulator region so that the effective mobility of the conduction path could be reduced.

Fig. 9 shows how seriously the $V_{G}$-dependent mobility affects the charge-based $R_{c}$ model. Here, the TLM-extracted $R_{c}$ values are plotted as a function of $\left|V_{G}-V_{T}\right|$. As expected, $R_{c}$ decreases rapidly with increasing $\left|V_{G}-V_{T}\right|$. We simply replaced $\mu$ in (34) by (38) and calculated $R_{c}$ to compare with the experimental curve. Note that the entire curve in Fig. 8 cannot be modeled by a simple analytical expression, so we only took the trap-dominated regime $\left(\left|V_{G}-V_{T}\right|<30 \mathrm{~V}\right)$ for the analysis in Fig. 9. As emphasized in Section IV-B, $V_{G}$ and $E_{b}$ are the foremost parameters that govern $R_{c}$. Therefore, by optimizing the TLM-extracted curve with the model, we could effectively estimate the injection barrier $E_{b}$ at the Au/pentacene interface in the fabricated OFETs. One can see that the model-calculated curve is in very good agreement with the experimental curve with $E_{b}$ of $0.38 \mathrm{eV}$. When we calculated $R_{c}$ with a constant mobility of $0.2 \mathrm{~cm}^{2} / \mathrm{V} \cdot \mathrm{s}$, the model could not accurately predict the experimental results.

In the literature, there has been a question about how sometimes $R_{c}$ is less sensitive to $V_{G}$ or even assumed to be constant [31], [12] while more often $R_{c}$ is strongly dependent on $V_{G}$. For coplanar OFETs, the whole picture of this study can suggest two answers: First, the injection barrier $E_{b}$ plays an important role on the dependence of $R_{c}$ on $V_{G}$ (Fig. 5) thus the material combination of the metal and the semiconductor is basically crucial [32]. Second, $V_{G}$-dependent mobility can either intensify or diminish the slope of an $R_{c}$ versus $V_{G}$ curve, because $\mu$ can either increase or decrease (or even remain stable) with $V_{G}$, depending on various geometrical and/or electrical mechanisms [22], [29], [33], [34].

\section{CONCLUSION}

In this study, a physical model for coplanar OFETs has been presented. Starting from the coupled Poisson's and transport equations, we could formulate an approximate analytical model for the charge distribution inside an organic semiconductor. The distribution functions were inspired by the classical theory of metal/insulator contacts because unintentionally doped organic 
semiconductors are characterized by very low thermal carrier density. The origin of the contact resistance was attributed to a low-carrier-density zone at the source/channel edge. An equation for the contact resistance was suggested and the model could explain the gate-voltage dependence of the contact resistance. Pentacene-based OFETs were fabricated and analyzed by the transmission-line method. It was found that, in most cases, the mobility is strongly dependent on the gate-voltage and this effect can further complicate the dependence of the contact resistance on the gate-voltage. We believe that this model can be widely applied for interpreting and predicting the behavior of coplanar OFETs and will deepen the fundamental understanding of the device physics of organic devices.

\section{ACKNOWLEDGMENT}

C. H. Kim would like to thank the Vice Presidency for External Relations (DRE) in Ecole Polytechnique for the Ph.D. fellowship.

\section{REFERENCES}

[1] D. Braga and G. Horowitz, "High-performance organic field-effect transistors," Adv. Mater, vol. 21, no. 14-15, pp. 1473-1486, Apr. 2009.

[2] A. Wan, J. Hwang, F. Amy, and A. Kahn, "Impact of electrode contamination on the $\alpha$-NPD/Au hole injection barrier," Org. Electron., vol. 6, no. 1, pp. 47-54, Feb. 2005.

[3] S. Braun, W. R. Salaneck, and M. Fahlman, "Energy-level alignment at organic/metal and organic/organic interfaces," Adv. Mater, vol. 21, no. 14-15, pp. 1450-1472, Apr. 2009.

[4] R. A. Street and A. Salleo, "Contact effects in polymer transistors," Appl. Phys. Lett., vol. 81, no. 15, pp. 2887-2889, Oct. 2002.

[5] K. P. Puntambekar, P. V. Pesavento, and C. D. Frisbie, "Surface potential profiling and contact resistance measurements on operating pentacene thin-film transistors by Kelvin probe force microscopy," Appl. Phys. Lett., vol. 83, no. 26, pp. 5539-5541, Dec. 2003.

[6] P. V. Pesavento, R. J. Chesterfield, C. R. Newman, and C. D. Frisbie, "Gated four-probe measurements on pentacene thin-film transistors: Contact resistance as a function of gate voltage and temperature," J. Appl. Phys., vol. 96, no. 12, pp. 7312-7324, Dec. 2004.

[7] C. Bock, D. V. Pham, U. Kunze, D. Kafer, G. Witte, and C. Woll, "Improved morphology and charge carrier injection in pentacene field-effect transistors with thiol-treated electrodes," J. Appl. Phys., vol. 100, no. 11, p. 114517, Dec. 2006.

[8] S. D. Wang, Y. Yan, and K. Tsukagoshi, "Understanding contact behavior in organic thin film transistors," Appl. Phys. Lett., vol. 97, no. 6, p. 063307, Aug. 2010.

[9] C.-W. Sohn, T.-U. Rim, G.-B. Choi, and Y.-H. Jeong, "Analysis of contact effects in inverted-staggered organic thin-film transistors based on anisotropic conduction,” IEEE Trans. Electron Devices, vol. 57, no. 5, pp. 986-994, May. 2010.

[10] T. J. Richards and H. Sirringhaus, "Analysis of the contact resistance in staggered, top-gate organic field-effect transistors," J. Appl. Phys., vol. 102, no. 9, p. 094510, Nov. 2007

[11] V. Vinciguerra, M. L. Rosa, D. Nicolosi, G. Sicurella, and L. Occhipinti, "Modeling the gate bias dependence of contact resistance in staggered polycrystalline organic thin film transistors," Org. Electron., vol. 10, no. 6, pp. 1074-1081, Sep. 2009.

[12] C. H. Kim, Y. Bonnassieux, and G. Horowitz, "Fundamental benefits of the staggered geometry for organic field-effect transistors," IEEE Electron Device Lett., vol. 32, no. 9, pp. 1302-1304, Sep. 2011.

[13] C. H. Kim, O. Yaghmazadeh, D. Tondelier, Y. B. Jeong, Y. Bonnassieux, and G. Horowitz, "Capacitive behavior of pentacene-based diodes: Quasistatic dielectric constant and dielectric strength," J. Appl. Phys., vol. 109, no. 8, p. 083710, Apr. 2011.

[14] N. F. Mott and R. W. Gurney, Electronic Processes in Ionic Crystals, 2nd ed. Oxford: Oxford University Press, 1940.

[15] S. M. Skinner, "Diffusion, static charges, and the conduction of electricity in nonmetallic solids by a single charge carrier. I. electric charges in plastics and insulating materials," J. Appl. Phys., vol. 26, no. 5, pp. 498-508, May 1955.

[16] S. M. Sze and K. K. Ng, Physics of Semiconductor Devices, 3rd ed. New York: Wiley, 2007.

[17] H. Klauk, G. Schmid, W. Radlik, W. Weber, L. Zhou, C. D. Sheraw, J. A. Nichols, and T. N. Jackson, "Contact resistance in organic thin film transistors," Solid-State Electron., vol. 47, no. 2, pp. 297-301, Feb. 2003.

[18] D. J. Gundlach, L. Zhou, J. A. Nichols, T. N. Jackson, P. V. Necliudov, and M. S. Shur, "An experimental study of contact effects in organic thin film transistors," J. Appl. Phys., vol. 100, no. 2, p. 024509, Jul. 2006.

[19] G. B. Blanchet, C. R. Fincher, M. Lefenfeld, and J. A. Rogers, "Contact resistance in organic thin film transistors," Appl. Phys. Lett., vol. 84, no. 2, pp. 296-298, Jan. 2004

[20] S. H. Kim, S. H. Lee, and J. Jang, "High-performance n-channel organic thin-film transistor for CMOS circuits using electron-donating self-assembled layer," IEEE Electron Device Lett., vol. 31, no. 9, pp. 1044-1046, Sep. 2010.

[21] C. Goldmann, S. Haas, C. Krellner, K. P. Pernstich, D. J. Gundlach, and B. Batlogg, "Hole mobility in organic single crystals measured by a "flip-crystal" field-effect technique," J. Appl. Phys., vol. 96, no. 4, pp. 2080-2086, Aug. 2004.

[22] M. Mottaghi and G. Horowitz, "Field-induced mobility degradation in pentacene thin-film transistors," Org. Electron., vol. 7, no. 6, pp. 528-536, Dec. 2006.

[23] S. Luan and G. W. Neudeck, "An experimental study of the source/drain parasitic resistance effects in amorphous silicon thin film transistors," J. Appl. Phys., vol. 72, no. 2, pp. 766-772, Jul. 1992.

[24] J. Zaumseil, K. W. Baldwin, and J. A. Rogers, "Contact resistance in organic transistors that use source and drain electrodes formed by soft contact lamination,” J. Appl. Phys., vol. 93, no. 10, pp. 6117-6124, May 2003.

[25] D. Boudinet, G. L. Blevennec, C. Serbutoviez, J.-M. Verilhac, H. Yan, and G. Horowitz, "Contact resistance and threshold voltage extraction in n-channel organic thin film transistors on plastic substrates," J. Appl. Phys., vol. 105, no. 8, p. 084510, Apr. 2009.

[26] G. Horowitz, P. Lang, M. Mottaghi, and H. Aubin, "Extracting parameters from the current-voltage characteristics of organic field-effect transistors," Adv. Funct. Mater, vol. 14, no. 11, pp. 1069-1074, Nov. 2004.

[27] S. Verlaak, V. Arkhipov, and P. Heremans, "Modeling of transport in polycrystalline organic semiconductor films," Appl. Phys. Lett., vol. 82, no. 5, pp. 745-747, Feb. 2003.

[28] A. Bolognesi, M. Berliocchi, M. Manenti, A. D. Carlo, P. Lugli, K. Lmimouni, and C. Dufour, "Effects of grain boundaries, field-dependent mobility, and interface trap states on the electrical characteristics of pentacene TFT," IEEE Trans. Electron Devices, vol. 51, no. 12, pp. 1997-2003, Dec. 2004.

[29] G. Horowitz, M. E. Hajlaoui, and R. Hajlaoui, "Temperature and gate voltage dependence of hole mobility in polycrystalline oligothiophene thin film transistors," J. Appl. Phys., vol. 87, no. 9, pp. 4456-4463, May 2000.

[30] W. L. Kalb and B. Batlogg, "Calculating the trap density of states in organic field-effect transistors from experiment: A comparison of different methods," Phys. Rev. B, Condens. Matter, vol. 81, no. 3, p. 035327, Jan. 2010.

[31] D. Braga and G. Horowitz, "Subthreshold regime in rubrene single-crystal organic transistors," Appl. Phys. A, Mater. Sci. Process., vol. 95, no. 1, pp. 193-201, Apr. 2009. 
[32] A. Kahn, N. Koch, and W. Gao, "Electronic structure and electrical properties of interfaces between metals and $\pi$-conjugated molecular films," $J$. Polym. Sci. Pt. B, Polym. Phys., vol. 41, no. 21, pp. 2529-2548, Nov. 2003.

[33] J. Takeya, M. Yamagishi, Y. Tominari, R. Hirahara, Y. Nakazawa, T. Nishikawa, T. Kawase, T. Shimoda, and S. Ogawa, "Very high-mobility organic single-crystal transistors with in-crystal conduction channels," Appl. Phys. Lett., vol. 90, no. 10, p. 102120, Mar. 2007.

[34] J. A. Merlo, C. R. Newman, C. P. Gerlach, T. W. Kelley, D. V. Muyres, S. E. Fritz, M. F. Toney, and C. D. Frisbie, "p-channel organic semiconductors based on hybrid acene-thiophene molecules for thin-film transistor applications," J. Am. Chem. Soc., vol. 127, no. 11, pp. 3997-4009, Mar. 2005.

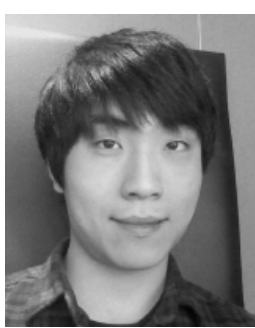

Chang Hyun Kim (S'11) received the dual M.Sc. degree from Kyung Hee University, Seoul, Korea, and Ecole Polytechnique, Palaiseau, France, in 2010. He is currently working toward the Ph.D. degree at Ecole Polytechnique.

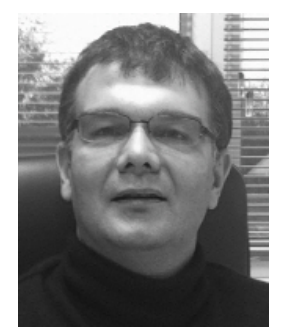

Yvan Bonnassieux received the Ph.D. degree from ENS, Cachan, France, in 1998.

$\mathrm{He}$ is currently an Assistant Professor with Ecole Polytechnique, Palaiseau, France, where he is the Head of the Organic Electronics Research Team.

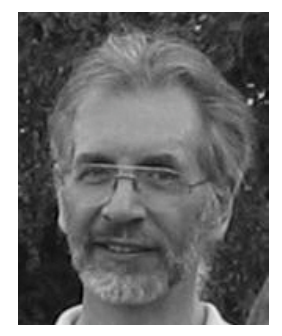

Gilles Horowitz received the Ph.D. degree from University Paris Diderot, Paris, France, in 1975.

He is Senior Research Fellow with the Centre National de la Recherche Scientifique (CNRS) ans is currently working at Ecole Polytechnique, Palaiseau, France. 